\title{
Characteristics of Carbohydrate Metabolism, Grain Quality and Yield Provide Insights into Rice-Tartary Buckwheat Improvement
}

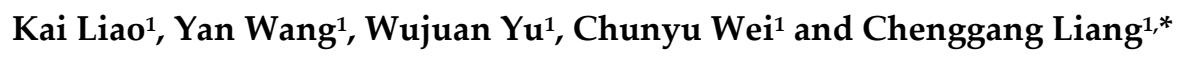 \\ 1 Research Center of Buckwheat Industry Technology, Guizhou Normal University, Guiyang 550001 China; \\ 475880336@qq.com (K.L.); yangwanguf@163.com (Y.L.); 1966855821@qq.com (C.W.); jesselcg@163.com (C.L.) \\ * Correspondence: jesselcg@163.com; Tel.: +86-0851-86780646
}

\begin{abstract}
Rice-Tartary buckwheat is a special type of Tartary buckwheat with easy hulling thin shell. The local rice-Tartary buckwheat 'cv. Xiaomiqiao' (XMQ) was used to explore the characteristics of photosynthesis, starch synthesis, yield and quality, as compared to Tartary buckwheat 'cv. Jinqiao 2'(JQ2). XMQ showed significantly lower values of net photosynthetic rate, ADPGase activity and starch synthase activity at one or more periods during grain filling, as compared to that of JQ2. The effective branch number and grain number per plant of XMQ were similar to that of JQ2 regardless of different years, but the 1000-grain weight was significantly lighter that resulted in the significantly lower yield. XMQ accumulated similar contents of starch (amylose, amylopectin) and protein (glutelin, albumin, prolamin, globulin) to that of JQ2, but exhibited significantly lower flavonoid content. The values of peak viscosity and hot paste viscosity in XMQ were similar to that of JQ2, but the values of cool paste viscosity, breakdown and setback were significantly lower. In conclusion, $\mathrm{XMQ}$ showed low yield due to the insufficient of photosynthesis and starch synthesis. XMQ exhibited high nutritional quality as well as normal Tartary buckwheat and stored $18.88 \mathrm{mg} \mathrm{g}^{-1}$ of flavonoid, that might be consumed as daily diet. The present results will provide a basis for food processing and breeding of rice-Tartary buckwheat.
\end{abstract}

Keywords: net photosynthetic rate; key enzymes activity; starch pasting viscosity; yield; quality

\section{Introduction}

Tartary buckwheat (Fagopyrum tataricum (L.) Gaertn) is a functional coarse cereal belongs to Fagopyrum Mill[1-3]. The high levels of bioactive phytochemicals in Tartary buckwheat show many health benefits, such as anti-hyperglycemia, anti-hypertension and anti-hyperlipidemia[2, 4-6]. However, Tartary buckwheat is rare consumed as daily diet around the world due to the bitter taste of flour for cooking like wheat or the barrier of hulling for cooking like rice[7]. The high level of rutin in Tartary buckwheat flour is quickly hydrolyzes to quercetin that is proved to be response to the bitter taste, but the rutin in Tartary buckwheat dehulled whole groats is rarely degraded when cooked[8-9]. The normal Tartary buckwheat is generally used to process functional foods, such as tea, pasta and bakery products, due to the thick shell that is difficult to hull. In Yunnan province, a local variety ' $\mathrm{cv}$. Xiaomiqiao' $(\mathrm{XMQ})$, known as the first cultivar of rice-Tartary buckwheat, is easily hulled and cooked like rice due to the non- adhering thin shell[8, 10, 11]. Many Asian like to cook rice mixing with coarse cereals to achieve balanced nutrition and health benefits. Therefore, it is acceptable to consume rice-Tartary buckwheat as daily diet in rice eating population[8, 10, 12].

Tartary buckwheat is regards as a low yield cereal that is typically cultivated in Southwest China. The foundational research of Tartary buckwheat is seriously lagged behind mass cereals. It is proposed that the indefinite inflorescence in buckwheat leads to the confusion of carbohydrate metabolism that further affects the foundation of yield[13-15]. Till now, the related report about the plant growth, yield and quality of rice-Tartary buckwheat is deficient. Crossbreeding of rice-Tartary 
buckwheat has been firstly reported by Wang and Campbell, and it is proposed that the tiny flowers and low seeds fertility lead to the difficulty of hybridization[10]. Therefore, it is crucial to make certain the agronomic and quality characteristics of rice-Tartary buckwheat for further crossbreeding using the present thin shell germplasm resource. In this study, the rice-Tartary buckwheat was firstly used to explore the characteristics of photosynthesis, starch synthesis, quality and yield, as compared to normal Tartary buckwheat 'cv. Jinqiao 2'(JQ2). The present results will provide a basis for high yield and quality breeding of rice-Tartary buckwheat.

\section{Materials and Methods}

\subsection{Plant materials}

The rice-Tartary buckwheat variety 'cv. Xiaomiqiao' $(\mathrm{XMQ})$ was planted in Experiment Station of the Research Center of Buckwheat Engineering Technology in Guizhou Province (1146m, 26 50', $106^{\circ} 58^{\prime}$ ) during autumn growth season of 2017 and 2018, as compared to the normal Tartary buckwheat variety 'Jinqiao2'(JQ2).

2.2 Plant growth and sampling

A randomized block design with three replications was performed in this study. The area for each test plot was $10 \mathrm{~m}^{2}(2 \mathrm{~m} \times 5 \mathrm{~m})$ with $33 \mathrm{~cm}$ of row width, and the planting density was approximately 1,000,000 plants per hectare. The soil in test pot was yellow loam that contained 12.15 $\mathrm{g} \cdot \mathrm{kg}^{-1}$ organic matter, $0.81 \mathrm{~g} \cdot \mathrm{kg}^{-1}$ total nitrogen, $96.37 \mathrm{mg} \cdot \mathrm{kg}^{-1}$ alkaline nitrogen, $12.42 \mathrm{mg} \cdot \mathrm{kg}^{-1}$ valid phosphorus, and $118.57 \mathrm{mg} \cdot \mathrm{kg}^{-1}$ valid potassium. The fertilizer at rates of $60 \mathrm{~kg} \mathrm{~N} \mathrm{ha-1}, 60 \mathrm{~kg} \mathrm{P}_{2} \mathrm{O}_{5}$ $\mathrm{ha}^{-1}$, and $30 \mathrm{~kg} \mathrm{~K}_{2} \mathrm{O} \mathrm{ha-1}$ was applied as base fertilizer in each plot before sowing. The consistent management measures were conducted and plants grew naturally with no significant incidence of weeds, pests or disease was observed.

To identify the key carbohydrates levels and enzymes activities involving starch synthesis, approximately 300-400 grains of 30-50 plants with similar developmental stage from each test plot were labeled and approximately 100 grains from 10 plants were respectively sampled at early grain filling stage(early-GFS), mid-GFS and late-GFS in 2017. Meanwhile, the top fully matured leaf of 10 plants was labeled and used for photosynthetic characterization.

To identify the agronomic traits, 12 plants from each text plot were sampled at harvest in 2017 and 2018. The plant height, effective branching number, grain number per plant, 1000-grain weight, and yield per plant were measured. The rest plants of each plot were harvested for the measurement of yield. Then, the grains of plants were artificially hulled and grinded to sieve with the 100-mesh for quality analysis.

\subsection{Experimental methods}

\subsubsection{Assay of Starch}

A $100 \mathrm{mg}$ of sample was eluted to remove soluble sugars for three times using $2 \mathrm{~mL}$ of $95 \%$ ethanol for assay of starch. The details for amylose and amylopectin measurement were performed according to the methods of Liang[16]. Starch content was calculated by amylose content plus amylopectin content.

\subsubsection{Assay of Protein}

A $100 \mathrm{mg}$ of sample was used to extract albumin, glutelin, prolamine and globulin by adding 5 $\mathrm{mL}$ of $10 \mathrm{mmol} / \mathrm{L} \mathrm{Tris-HCl}$ (pH 7.5), $5 \mathrm{~mL}$ of $0.5 \mathrm{~mol} / \mathrm{L} \mathrm{NaCl}, 5 \mathrm{~mL}$ of $60 \%$ normal propyl alcohol and $5 \mathrm{~mL}$ of mixed liquor (containing $0.5 \%$ sodium tartrate, $0.24 \%$ copper sulfate, $1.68 \% \mathrm{KOH}$ and $50 \%$ normal propyl alcohol), respectively for three times. The details for albumin, glutelin, prolamine and globulin measurement were performed according to the methods of Wang et al[17]. Total protein content was calculated by the sum of albumin, glutelin, prolamine and globulin.

\subsubsection{Assay of Flavonoid}

A $100 \mathrm{mg}$ of sample was used to extract flavonoid by adding $2 \mathrm{~mL}$ of $80 \%$ ethanol for hot bath at $70{ }^{\circ} \mathrm{C}$ for $5 \mathrm{~h}$ and ultrasonic extraction for $10 \mathrm{~min}$. The details for flavonoid measurement were performed according to the methods of Wang et al[17].

\subsubsection{Assay of RVA pasting parameter}


A 3,000 $\mathrm{mg}$ of sample and $25 \mathrm{~mL}$ distilled water were added into tube and detected by rapid visco analyser (RVA, Newport Scientific, Australia). Parameter was performed according to Xu et al[18].Then, the values of peak viscosity (PV), hot paste viscosity (HPV), cool paste viscosity (CPV), breakdown (BD), setback (SB), consistency (CS) and pasting temperature (PT) were analyzed by software kit of Thermal Cycle Window according to Tone et al[19].

\subsection{Statistical analyses}

Microsoft Excel 2003 (Microsoft Corporation, Santa Rosa, California, USA) was used for data statistics and analysis. SPSS Statistics 17.0 (IBM, Chicago, IL, USA) was used for data variance analysis. The difference between two cultivars were performed by t-test $(\mathrm{p}<0.05)$.

\section{Results}

\subsection{Carbohydrate metablism}

To identify the carbohydrate metabolism of rice-Tartary buckwheat, the net photosynthetic rate and key enzymes activities involving starch synthesis were investigated. As shown in Table 1, the net photosynthetic rate of the top fully matured leaf in XMQ were significantly lower at early-GFS and mid-GFS, as compared to that of JQ2. No statistic difference of net photosynthetic rate was detected at late-GFS between XMQ and JQ2 due to the leaf senescence that caused the rapid decrease of photosynthesis. By contrast, there was little difference of stomatal conductance and water use efficiency in the top full expanded leaf between XMQ and JQ2 during grain filling.

Table 1. Photosynthetic characteristics in leaves of Tartary buckwheat during grain filling

\begin{tabular}{ccccccc}
\hline \multirow{2}{*}{ Period } & \multicolumn{2}{c}{$\begin{array}{c}\text { Net photosynthetic rate } \\
\boldsymbol{\mu m o l} /\left(\mathbf{m}^{2} \cdot \mathbf{s}\right)\end{array}$} & \multicolumn{2}{c}{$\begin{array}{c}\text { Stomatal conductance } \\
\boldsymbol{\mu m o l} /\left(\mathbf{m}^{2} \cdot \mathbf{s}\right)\end{array}$} & \multicolumn{2}{c}{$\begin{array}{c}\text { Water use efficiency } \\
(\%)\end{array}$} \\
\cline { 2 - 7 } & $\mathbf{X M Q}$ & $\mathrm{JQ2}$ & $\mathbf{X M Q}$ & $\mathrm{JQ2}$ & $\mathrm{XMQ}$ & $\mathrm{JQ2}$ \\
\hline Early-GFS & $9.38 \pm 0.18$ & $10.38 \pm 0.59^{*}$ & $4.96 \pm 1.37$ & $5.26 \pm 1.37$ & $0.76 \pm 0.21$ & $0.96 \pm 0.42$ \\
Mid-GFS & $9.43 \pm 0.32$ & $10.38 \pm 0.42^{*}$ & $3.63 \pm 1.08$ & $3.21 \pm 1.02$ & $0.89 \pm 0.10$ & $0.97 \pm 0.35$ \\
Late-GFS & $1.52 \pm 0.30$ & $1.28 \pm 0.26$ & $6.00 \pm 1.80$ & $6.00 \pm 2.14$ & $0.62 \pm 0.15$ & $0.52 \pm 0.19$ \\
\hline
\end{tabular}

* represents the significant difference $(P<0.05)$ betweenXMQ and JQ2. The same as below.

As shown in Figure 1, the starch levels of grains in XMQ were slightly lower than JQ2 during grain filling without statistic difference. In addition, no statistic difference of sucrose and glucose levels were detected between XMQ and JQ2 during grain filling. However, the activities of ADPGase at mid-GFS and SS at late-GFS in grains of XMQ were significantly lower than that of JQ2 (Figure 2).

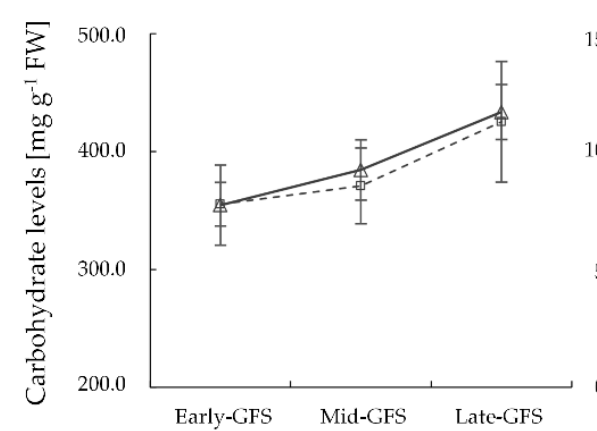

(a)

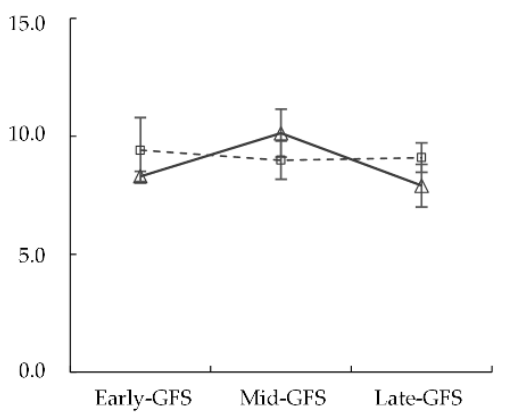

(b)

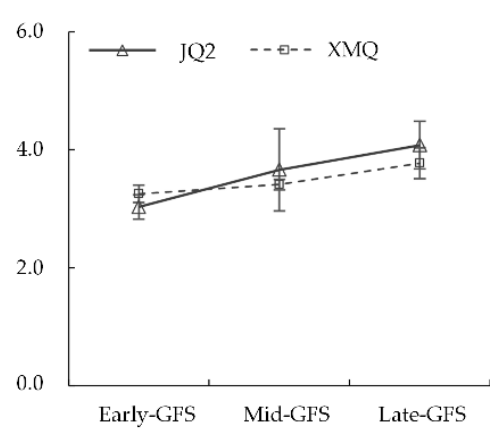

(c)

Figure 1. The levels of starch(a), sucrose (b) and glucose (c) in grains of Tartary buckwheat during grain filling. 


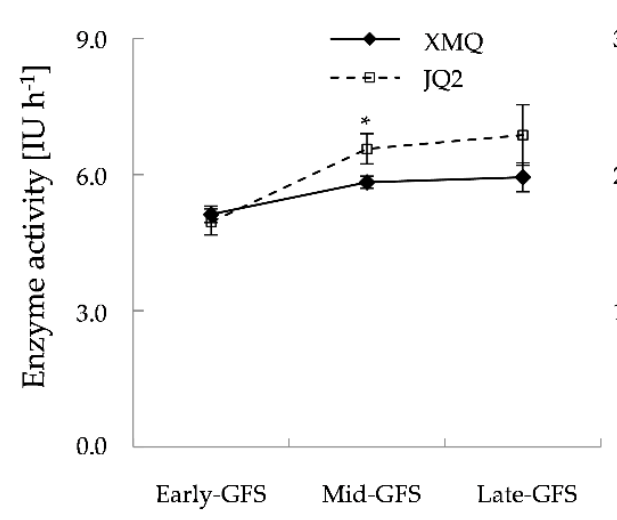

(a)

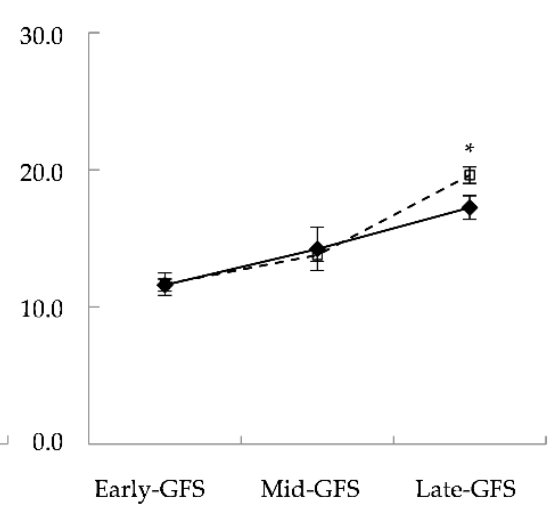

(b)

Figure 2. The activities of ADPGase(a) and SS (b) in grains of Tartary buckwheat during grain filling.

\subsection{Agronomic traits}

To identify the plant growth and yield of rice-Tartary buckwheat, the agronomic traits were investigate at harvest in 2017 and 2018. As shown in table 2, the plant height of XMQ was significantly higher than JQ2, but no statistic difference of effective branch number was found. The number of grains per plant in XMQ was slightly lower than JQ2 without statistic difference, but the grain weight per plant was significantly lower than JQ2 due to the significantly lower 1000-grain weight. The yield of XMQ were significantly lower than JQ2 in 2017 and 2018.

Table 2. Agronomic traits of Tartary buckwheat.

\begin{tabular}{|c|c|c|c|c|c|c|c|}
\hline Year & Cultivar & $\begin{array}{l}\text { Plant height } \\
\text { (cm) }\end{array}$ & $\begin{array}{c}\text { Effective } \\
\text { branch } \\
\text { number }\end{array}$ & $\begin{array}{c}\text { Number of } \\
\text { grains per } \\
\text { plant }\end{array}$ & $\begin{array}{l}\text { 1000- grain } \\
\text { weight } \\
\text { (g) }\end{array}$ & $\begin{array}{c}\text { Grain } \\
\text { weight } \\
\text { per plant } \\
\text { (g) }\end{array}$ & $\begin{array}{l}\text { Yield } \\
\left(\mathrm{t} / \mathrm{hm}^{2}\right)\end{array}$ \\
\hline \multirow[t]{2}{*}{2017} & & & & & & $3.05 \pm$ & \\
\hline & JQ2 & & & 8 & 20.3 & 4.42 & 1.36 \\
\hline \multirow[t]{2}{*}{2018} & XMQ & $122.22 \pm 7.03$ & $7.11 \pm 0.19$ & $203.67 \pm 45.00$ & $16.35 \pm 0.28$ & $3.29 \pm 0.14$ & $0.73 \pm 0.10$ \\
\hline & JQ2 & $101.56 \pm 5.80^{* *}$ & $7.11 \pm 0.51$ & $214.11 \pm 19.45$ & $20.62 \pm 0.36^{* *}$ & $4.33 \pm 0.26^{* *}$ & $1.28 \pm 0.13^{* *}$ \\
\hline
\end{tabular}

\subsection{Quality traits}

To identify the quality of rice-Tartary buckwheat, the starch, protein, flavonoid and RVA pasting parameters were detected. As shown in Table 3, the compositions of starch and protein in XMQ were similar to that of JQ2, noticing that no statistic differences of amylopectin, amylose, glutelin, albumin, prolamin and globulin were found between XMQ and JQ2. But, the flavonoid content in $\mathrm{XMQ}$ was $18.88 \mathrm{mg} / \mathrm{g}$, that was significantly lower than JQ2. From table 4, no statistic differences of PV and HPV were detected between XMQ and JQ2, whereas the BD, CPV and SB of XMQ were significantly lower than that of JQ2.

Table 3. The major storage materials in grains of Tartary buckwheat.

\begin{tabular}{cccccccc} 
Cultivar & $\begin{array}{c}\text { Amylopectin } \\
(\mathrm{mg} / \mathrm{g})\end{array}$ & $\begin{array}{c}\text { Amylose } \\
(\mathrm{mg} / \mathrm{g})\end{array}$ & $\begin{array}{c}\text { Glutelin } \\
(\mathrm{mg} / \mathrm{g})\end{array}$ & $\begin{array}{c}\text { Albumin } \\
(\mathrm{mg} / \mathrm{g})\end{array}$ & $\begin{array}{c}\text { Prolamin } \\
(\mathrm{mg} / \mathrm{g})\end{array}$ & $\begin{array}{c}\text { Globulin } \\
(\mathrm{mg} / \mathrm{g})\end{array}$ & $\begin{array}{c}\text { Flavonoid } \\
(\mathrm{mg} / \mathrm{g})\end{array}$ \\
\hline $\mathrm{XMQ}$ & $397.39 \pm 26.26$ & $153.56 \pm 7.40$ & $36.8 \pm 4.39$ & $23.42 \pm 2.78$ & $14.15 \pm 1.39$ & $3.22 \pm 0.04$ & $18.88 \pm 0.48$ \\
$\mathrm{JQ} 2$ & $420.01 \pm 22.80$ & $160.82 \pm 6.27$ & $39.6 \pm 3.72$ & $25.21 \pm 1.47$ & $16.09 \pm 1.91$ & $3.43 \pm 0.14$ & $21.15 \pm 0.39$ \\
\hline
\end{tabular}


Table 4. The RVA pasting parameter of Tartary buckwheat.

\begin{tabular}{ccccccc}
\hline \multirow{2}{*}{ Cultivar } & PV & HPV & BD & CPV & SB & PT \\
& & & & & & \\
\hline XMQ & $128.80 \pm 0.53$ & $128.42 \pm 1.06$ & $0.38 \pm 1.59$ & $217.13 \pm 4.07$ & $88.33 \pm 3.54$ & $61.2 \pm 0.00$ \\
JQ2 & $131.38 \pm 4.42$ & $127.54 \pm 5.00$ & $3.84 \pm 0.59^{*}$ & $231.92 \pm 2.00^{*}$ & $100.54 \pm 2.42^{*}$ & $64.2 \pm 0.00^{*}$ \\
\hline
\end{tabular}

\section{Discussion}

\subsection{Yield assessment for improvement of rice-Tartary buckwheat}

Tartary buckwheat is typically planted in cold highland area of Southwest China. The cultivated area is progressively decreased during the last 30 years due to the low yield character[16, 20]. The high-yield breeding work of Tartary buckwheat has been focused in China only for a few years[21]. The thick shell Tartary buckwheat 'JQ2' has been proved as a high yield cultivar for planting in Guizhou Province[16]. In this study, the thin shell rice-Tartary buckwheat ' $X M Q^{\prime}$ showed significant lower yield than JQ2 in two test years. Therefore, it was crucial to improve the yield of rice-Tartary buckwheat. XMQ showed similar effective branch number and grain number per plant to JQ2, but exhibited significantly lower 1000-grain weight. In addition, XMQ showed significantly higher plant height that increased the risk of lodging. Therefore, the improvement of grain size and plant height were proposed in high yield breeding of rice-Tartary buckwheat.

Tartary buckwheat is a typical crop with short growth period, thus the contradiction between vegetative growth and reproductive growth is prominent[15]. XMQ showed significantly lower net photosynthetic rate at early-GFS and mid-GFS, and significantly lower activities of ADPGase at mid-GFS and SS at late-GFS, as compared to that of JQ2. Thus, the yield of XMQ was limited by the insufficient of leaf photosynthetic assimilation for starch synthesis and accumulation in seed. Although XMQ showed similar levels of glucose, sucrose and starch to JQ2 during grain filling, it was easy to calculate that XMQ accumulated significantly lower carbohydrates in grains per plant. Therefore, the improvement of carbohydrate assimilation for starch synthesis was proposed in high yield breeding of rice-Tartary buckwheat.

\subsection{Quality assessment for improvement of rice-Tartary buckwheat}

Starch is the dominant storage material that accounts for approximately 60 70\% of Tartary buckwheat kernel. XMQ showed similar composition and content of starch in kernel, as compared to JQ2. Protein is the dominant nutritional material of Tartary buckwheat. Also, XMQ showed similar composition and content of protein in kernel, as compared to JQ2. RVA profile has been suggested as an assessment indicator for high quality breeding of rice. Generally, the rice with higher BD value and lower SB value has been suggested as good quality variety. In this study, the RVA profile was performed using Tartary buckwheat flours. Although XMQ showed significantly lower values of BD, CPV and SB than JQ2, the variation of values were small. In addition, the values of PV and HPV in XMQ were similar to JQ2. These results indicated that the composition and content of major storage materials in rice-Tartary buckwheat was consistent with normal Tartary buckwheat. Tartary buckwheat is a functional cereal rich in flavonoids and it has long been suggested as the source of rutin in daily diet[22]. XMQ is the well-known rice-Tartary buckwheat with non-adhering thin shell to process whole groats that avoids the degradation of rutin when cooked as daily $\operatorname{diet}[9]$. XMQ accumulated $18.88 \mathrm{mg} / \mathrm{g}$ of total flavonoid that was significantly lower than JQ2. Therefore, the improvement of flavonoid content was suggested in high quality breeding of rice-Tartary buckwheat. 


\section{Author Contributions:}

Conceptualization, C.L; investigation, K.L., W.Y., C.W. and Y.W.; data curation, Y.W.; writing-original draft preparation, K.L and Y.W.; writing - review and editing, C.L.; funding acquisition, C.L.

Funding: This research was funded by National Natural Science Foundation of China (31660366) and Natural Science Foundation of Guizhou Province(QKH CG[2019]4336, QKH ZC[2019]2298, QKH LH[2017]7359, QKH PT[2017]5726).

Acknowledgments: We thank PhD. Yan Lan for the technical support of RVA pasting parameter detection.

Conflicts of Interest: The authors declare no conflict of interest.

\section{References}

1. Hunt H. V.; Shang X.; Jones M.K. Buckwheat: a crop from outside the major Chinese domestication centres? A review of the archaeobotanical, palynological and genetic evidence[J]. Veget. Hist. Archaeobot. 2018, 27, 493-506

2. Tomotake H.; Kayashita J.; Kato N. Hypolipidemic activity of common (Fagopyrum esculentum Moench) and tartary (Fagopyrum tataricum Gaertn.) buckwheat. J Sci Food Agric. 2014, 95, 1963-1967.

3. Gao J.; Kreft I.; Chao G.; Wang Y.; Liu X.; Wang L.; Wang P.; Gao X.; Feng B. Tartary buckwheat (Fagopyrum tataricum Gaertn.) starch, a side product in functional food production, as a potential source of retrograded starch. Food Chem. 2016, 190, 552-558.

4. Choi J.Y.; Lee J.M.; Lee D.G.; Yoon Y.; Cho E.J.; Lee S. The n-Butanol Fraction and Rutin from Tartary Buckwheat Improve Cognition and Memory in an In Vivo Model of Amyloid- $\beta$-Induced Alzheimer's Disease. J Med Food. 2015, 18, 631-641.

5. Giménez-Bastida J.A.; Piskula M.K.; Zieliñski H. Recent Advances in Processing and Development of Buckwheat Derived Bakery and Non-Bakery Products-A Review. Polish J. Food Nutr. Sci. 2015, 65(1),9-20.

6. Morishita T.; Yamaguchi H.; Degi K. The contribution of polyphenols to antioxidative activity in common buckwheat and tartary buckwheat grain. Plant Prod. Sci. 2007, 10, 99-104.

7. Suzuki T.; Morishita T.; Mukasa Y.; Takigawa S.; Yokota S.; Ishiguro K.; Noda T. Breeding of 'Manten-Kirari', a non-bitter and trace-rutinosidase variety of Tartary buckwheat (Fagopyrum tataricum Gaertn.). Breeding Sci. 2014, 64,344-350.

8. Mukasa Y.; Suzuki T.; Honda Y (). Suitability of rice-Tartary buckwheat for crossbreeding and for utilization of rutin. Jpn. Agr. Res. Q. 2009, 43, 199-206.

9. Mukasa Y.; Suzuki T.; Morishita T. Rutin degradation in dehulled seeds of rice-Tartary buckwheat. Fagopyrum. 2009, 39-43.

10. Wang Y.; Campbell C.G. Tartary buckwheat breeding (Fagopyrum tataricum L. Gaertn.) through hybridization with its Rice-Tartary type. Euphytica, 2007, 156, 399-405.

11. Joshi B.K.; Bimb H.P.; Okuno K.; Rana C.B.; Vaidya M.L.; Sharma D.R. Bhate Phapar (rice Tartary buckwheat): diversity and uniqueness. Fagopyrum, 2009, 26, 31-37.

12. Chen Q.F.; Chen Q.J.; Shi T.X.; Huang K.F.; Liang L.B.; Rao Q.L.; Tang L.; Zhang Q.D. Inheritance of Tartary Buckwheat Thick Shell Character and Its Relationships with Yield Factors. Crops. 2015. 2, 27-31.

13. Tang Y.; Zhao G. A Source-Sink Ratio Change Affects the Fertilization and Fruiting of Tartary Buckwheat. Curr. Adv. Buckwheat Res. 1995, 519-523.

14. Xiang D.; Ma C.; Song Y.; Wu Q.; Wu X.; Sun Y.; Zhao G.; Wang Y. Post-Anthesis Photosynthetic Properties Provide Insights into Yield Potential of Tartary Buckwheat Cultivars. Agronomy. 2019, 9, 149.

15. Wang C.; She HZ.; Liu XB.; Hu D.; Yi Z.L. Effects of fertilization on leaf photosynthetic characteristics and grain yield in tartary buckwheat Yunqiao1. Photosynthetica, 2016, 55, 1-8.

16. Liang C.G.; Song Y.X.; Guo X.; Kong D.Z.; Wang Y.; Huang K.F. Characteristics of the Grain-filling Process and Starch Accumulation of High-yield Common Buckwheat 'cv. Fengtian 1' and Tartary Buckwheat 'cv. Jingqiao 2'. Cereal Res. Commun. 2016, 3:393-403.

17. Wang Y.; Liang C.G.; Shun Y.H.; Yu W.J.; Liao K.; Huang Y.W.; Shi T.X.; Meng Z.Y. The yield and quality of tartary buckwheat varieties and the response to low nitrogen. J. Guizhou Normal Univ (Nat. Sci.).2017, $35(6), 66-73$. 
18. Xu G.L.; Wang Y.; Liang C.G.; Jiang G.C.H.; Ding C.B.; Li T. Evaluations of cooking, eating and nutritional qualities of indica rice cultivars introduced from Sri Lanka. J. South Chin. Agri. Univ. 2016, 37(3), 37-45.

19. Tong C.; Chen Y.; Tang F.; Xu F.; Huang Y.; Chen H.; Bao J. Genetic diversity of amylose content and RVA pasting parameters in 20 rice accessions grown in Hainan, China. Food Chem. 2014, 161:239-245.

20. Giménez-Bastida, J.A.; Piskuła M.; Zieliński H. Recent advances in development of gluten-free buckwheat products. Trends Food Sci. Tech. 2015. 44(1), 58-65.

21. Chen Q.F. The status of buckwheat production and recent progresses of breeding on new type of cultivated buckwheat. J. Guizhou Normal Univ(Nat. Sci.).2019, 36(3), 1-7.

22. Fabjan N.; Rode J.; Iztok JK.; Wang Z.; Zhang Z.; Kreft I. Tartary Buckwheat (Fagopyrum tataricum Gaertn.) as a Source of Dietary Rutin and Quercitrin. J. Agri. Food Chem. 2003, 51, 6452-6455. 